\title{
Long-fibre reinforced polymer composites by 3D printing: influence of nature of reinforcement and processing parameters on mechanical performance
}

\author{
Francis Dantas, Kevin Couling and Gregory J. Gibbons* ${ }^{*}$
}

\begin{abstract}
The aim of this study was to identify the effect of material type (matrix and reinforcement) and process parameters, on the mechanical properties of 3D Printed long-fibre reinforced polymer composites manufactured using a commercial 3D Printer (Mark Two). The effect of matrix material (Onyx or polyamide), reinforcement type (Carbon, Kevlar ${ }^{\oplus}$, and HSHT glass), volume of reinforcement, and reinforcement lay-up orientation on both Ultimate Tensile Strength (UTS) and Flexural Modulus were investigated.

For Onyx, carbon fibre reinforcement offered the largest increase in both UTS and Flexural Modulus over unreinforced material (1228 $\pm 19 \%$ and $1114 \pm 6 \%$ respectively). Kevlar ${ }^{\oplus}$ and HSHT also provided improvements but these were less significant. Similarly, for Nylon, the UTS and Flexural Modulus were increased by $1431 \pm 56 \%$ and $1924 \pm 5 \%$ by the addition of carbon fibre reinforcement. Statistical analysis indicated that changing the number of layers of reinforcement had the largest impact on both UTS and Flexural Strength, and all parameters were statistically significant.
\end{abstract}

Keywords: Additive manufacturing, Polymer composites, Reinforcement, Tensile, Flexural

\section{Introduction}

Developments in composites compositions, and understanding the design and manufacturing using Additive Manufacturing (AM) is an important research field today [1], which can be seen from Wohler's Report 2016, that production of end use parts represented 51\% of the worldwide market using AM, with Material Extrusion being the most widely used method [2]; the reason being high demand of these materials in the aerospace and automobile sectors due to them being light-weight, having high static strength, good fatigue resistance and damage tolerance $[3,4]$. One of the most important applications is in light-weighting in the aerospace sector, one widely recognised being the main fuselage and the wings of the Boeing 787 being composite, and in total $50 \%$ of the material used in the aircraft is advanced

* Correspondence: g.j.gibbons@warwick.ac.uk

WMG, University of Warwick, Coventry CV4 7AL, UK composites. This reduces the maintenance requirements and cost, and increases the total reliability of the aircraft, reducing the weight by $20 \%$ [5]. In the automotive industry, the fuel efficiency can be increased by lightweighing cars, with the use of thermoplastic or thermoset composites instead of steel. A weight reduction of $55 \%$ was achieved for a conventional $9 \mathrm{~m}$ bus [6]. The energy absorption for some thermoplastic composites can be 7-8 times that of traditional steel [7], offering opportunities for improved crash protection.

There is a growing interest in the use of AM for the manufacture of composite components. The benefits of $\mathrm{AM}$, in offering flexibility in design (opening up the pathway to light-weight internal structuring and for part consolidation [8]), and tool-less manufacture (removing the cost burden and extensive lead-time for tooling, especially for low volume manufacture, as within the aerospace and niche vehicle land-transport sectors [9]). 
These capabilities make it a very attractive proposition for these sectors, but uptake has been limited to date due to the poor mechanical properties that are obtained from the polymers [10], with, until relatively recently, no capability for improving mechanical properties through their reinforcement using long or continuous fibres.

Recently, long fibre reinforcement of 3D Printed composites has been developed to a commercial offering, provided by, for example, MarkForged Inc. The technology, based on the Material Extrusion AM technology, where a filament of polymer is melted and extruded through a nozzle onto a planar platform, with addition of multiple layers forming the component. A continuous reinforcing filament is deposited onto, and embedded into, the deposited polymer tracks, thus allowing longfibre reinforcement of the polymer matrix [11].

The right balance of matrix and reinforcement materials in a polymer composite results in optimal tensile strength and stiffness for specific applications [12]. These properties are affected by the various build parameters during $\mathrm{AM}$, hence the selection of the right balance of composite components and optimal build parameters are equally important. Known mechanical properties are important to understand the reliability and the future performance of the part that is designed, and since the MarkForged technology is relatively new, there is little available mechanical performance data available. The aim of this paper is to characterise the mechanical performance of two common matrix materials employed in Material Extrusion (polyamide (PA) and Onxy (PA-carbon fibre composite) thermoplastics) with a range of fibre reinforcements (High Strength High Temperature Glass Fibre - HSHT (GF), Poly paraphenylene terephthalamide (Kevlar ${ }^{\circ}$ Fibre (KF), and Carbon Fibre (CF)), and processed using a range of build parameters (number of reinforcement layers and fibreplacement orientation). We aim to identify, through a statistical analysis of the material performance, the role of each material and build parameter choice on the mechanical response of the material. This will provide product designers with the tools to enable them to utilise this technology in designing products to satisfy engineering requirements.

AM is widely used for the fabrication of polymer components ranging from prototypes to end-use parts [13]. A wide range of AM technologies have been developed including Vat Polymerisation [14], Binder Jetting [15], Material Jetting [16] and Material Extrusion [17]. The latter is widely used due to its ease of use, low cost and ubiquity within the market-place [18]. The Material Extrusion process consists of a thermoplastic filament feed into a heated nozzle, where it is melted and deposited onto a planar platform under CNC (Computer Numerical Control). After the manufacture of one layer of the component, the platform lowers (or the nozzle is raised) by one-layer thickness (typically $0.05-0.2 \mathrm{~mm}$ ) and the next layer is deposited. This is continued until the product is complete.

Non-reinforced thermoplastics used in this process suffer from poor mechanical properties [10], which has limited the widespread uptake of this technology for end-use products. Some attempt has been made to improve properties, e.g. through the use of chopped fibre reinforcement, such as glass fibre and polypropylene (PP) fibre in PP matrix, showing 30 and 40\% improvement in Ultimate Tensile Strength (UTS) compared to pure PP [19], and $5 \mathrm{wt} \%$ of either vapour grown carbon fibres (VGCFs) and single wall carbon nanotubes (SWNTs) in an acrylonitrile butadiene styrene (ABS) providing a $18 \%$ and $31 \%$ improvement in UTS [20]. The larger challenge of incorporating continuous or long fibre reinforcement into AM polymer composites offers the opportunity for significantly larger improvements in mechanical properties. This has been achieved by a number of researchers, namely, Matsuzaki et al. [21], developed in-nozzle impregnation of carbon and other fibres in polylactic acid (PLA), with large increases in UTS and flexural modulus observed. Addition of reinforcing fibres into the deposited polymer layers was an approach taken by Mori et al. [22], with the embedding of Kevlar fibres into a nylon matrix using a desktop Material Extrusion system, improving strength and fatigue properties. Van Der Klift et al. [23] studied the mechanical behaviour of 6 layers of carbon fibre embedded in a polyamide matrix using a commercial Mark One AM system (Markforged Inc., USA), observing a 9x increase in UTS over the unreinforced material, with similar studies by Dickson et al. [12] extending the study to the effect of Kevlar reinforcement, with up to $6.3 \mathrm{x}$ increase in UTS and flexural strength observed.

There is thus a growing body of work in observing the effect of continuous fibre reinforcement of AM thermoplastic composites, providing evidence of useful and significant increases in mechanical properties. These studies though are, to date, lacking any use of experimental design accompanied by statistical analysis to elucidate the individual effects of material choice and build parameters on the mechanical response of the materials. This paper aims to provide product designers, and users of commercial continuous fibre AM technology, with a knowledge base capturing the impact and statistical significance of the choice of base material, the reinforcement material, and some key build parameters; allowing them to make informed decisions in the choice of materials and parameters to meet their design needs.

\section{Experimental}

Mechanical test samples were manufactured using a Mark Two Material Extrusion system (Markforged Inc., 
Table 1 Material properties for the matrix and reinforcement materials [26]

\begin{tabular}{llll}
\hline Material & UTS (MPa) & E (GPa) & Tensile Strain at Break (\%) \\
\hline Nylon & 36 & 1.4 & 150 \\
Onyx & 51 & 3.6 & 58 \\
GF & 600 & 21 & 3.9 \\
CF & 800 & 51 & 1.5 \\
KF & 610 & 26 & 2.7 \\
\hline
\end{tabular}

USA), using $\phi 0.4 \mathrm{~mm}$ nozzles at $270{ }^{\circ} \mathrm{C}, 0.1 \mathrm{~mm}$ layer thickness (to reduce the build cost [24] and to reduce the porosity and increase the mechanical strength [25]). The build platform was unheated. The base materials employed were nylon, a proprietary polyamide composition (Markforged Inc., USA), and Onyx, a proprietary short carbon fibre reinforced polyamide composite (Markforged Inc, USA). Both filaments were $1.75 \mathrm{~mm}$ diameter, and were kept in a dry box prior to, and during use. The reinforcing filaments used were glass Fibre (GF) $-0.3 \mathrm{~mm}$ bundles, carbon Fibre (CF) $-0.35 \mathrm{~mm}$ bundles, and Kevlar Fibre (KF) $-0.3 \mathrm{~mm}$ bundles, all supplied by MarkForged Inc. The material properties are provided in Table 1. A number of the available build parameters defining the structure of the samples (Fig. 1) were set at constant levels. The number of layers in the side walls, were set at 2 (default), although it is known that extra layers can increase part strength [24], the effect will be small, and, due to the limited width of the test samples, increasing the wall layer thickness will result in a decrease in reinforcement volume [27]. The number of floor and roof layers was both set to 4 (default). Zero concentric fibre rings were included. An infill density of $37 \%$ was employed (default), in triangular fill pattern. It is known to affect mechanical properties, with tensile strength increasing with density [24]) and $37 \%$ was selected as a good compromise between strength, build time (cost) and weight.

The parameters selected for variable analysis were base material, reinforcement material, number of reinforcement layers and reinforcement orientation. The values for the variables are given in Table 2. A unidirectional angle orientation has higher strength, but only in the direction of the fibre. However, to allow wider application in products where multi-directional strength is required, a bidirectional lay-up is desirable [28]. A standard full-factorial design of experiment (DoE) was employed to allow statistical variation of parameters to be assessed. A multilevel design was used, with 4 factors (three 2-level and one 3-level) and 5 replicates. The DoE structure is given in Table 2 (excluding replicants for brevity). Samples were also manufactured for un-reinforced Nylon and Onxy (5 of each) to provide baseline performance comparison.

The sample orientation was maintained constant for all parts (Fig. 2). This was chosen to minimise build time and allow maximum level of reinforcement to be introduced. Khatri [24] showed part orientation having only a small effect on part strength.

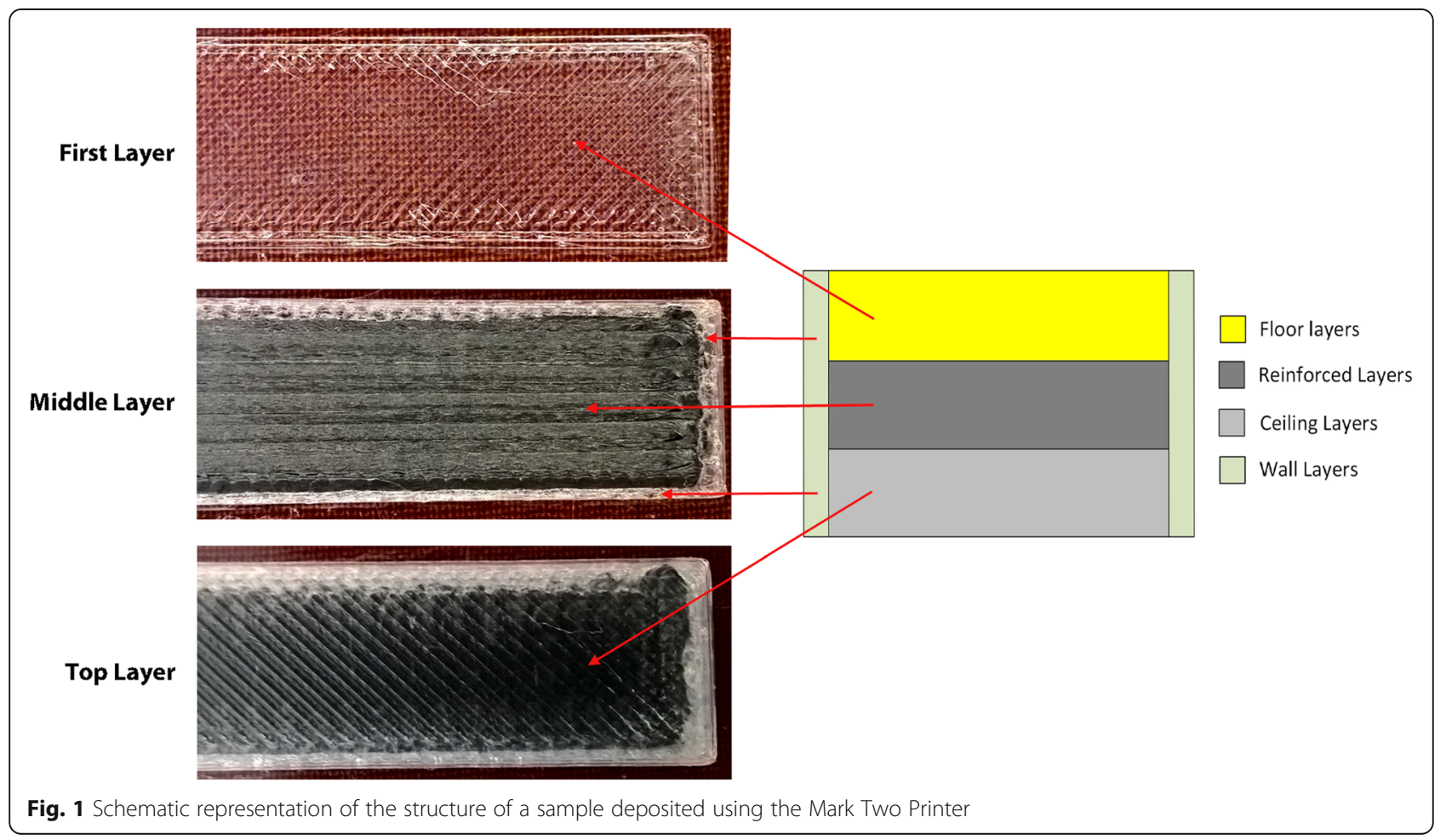


Table 2 Parameters Employed and Levels Set for Sample Manufacture

\begin{tabular}{|c|c|c|c|}
\hline Parameter & Number of Levels & Parameter Values & Justification \\
\hline Matrix Material (M) & 2 & Nylon, Onyx & Only two available \\
\hline Reinforcement Material (R) & 3 & GF, CF KF & Only three available \\
\hline Number of Fibre Layers (RL) & 2 & 4,12 & Allowable extremes for sample thickness \\
\hline Fibre Orientation (F) & 2 & $\left(0^{0}, 45^{\circ}\right),\left(0^{0}, 90^{\circ}\right)$ & Bi-directional for wider application of the materials \\
\hline Matrix & Reinforcement & Layers & Orientation \\
\hline Nylon & GF & 4 & 0,90 \\
\hline Nylon & GF & 12 & 0,90 \\
\hline Nylon & CF & 12 & 0,45 \\
\hline Nylon & CF & 4 & 0,90 \\
\hline Nylon & KF & 4 & 0,45 \\
\hline Nylon & KF & 12 & 0,90 \\
\hline Nylon & GF & 12 & 0,45 \\
\hline Nylon & KF & 12 & 0,45 \\
\hline Nylon & $\mathrm{KF}$ & 4 & 0,90 \\
\hline Nylon & KF & 12 & 0,90 \\
\hline Nylon & KF & 4 & 0,45 \\
\hline Nylon & GF & 4 & 0,90 \\
\hline Onyx & KF & 4 & 0,90 \\
\hline Onyx & GF & 12 & 0,90 \\
\hline Onyx & KF & 12 & 0,45 \\
\hline Onyx & GF & 4 & 0,45 \\
\hline Onyx & CF & 12 & 0,90 \\
\hline Onyx & CF & 12 & 0,45 \\
\hline Onyx & GF & 12 & 0,45 \\
\hline Onyx & $C F$ & 4 & 0,45 \\
\hline Onyx & GF & 4 & 0,45 \\
\hline Onyx & CF & 4 & 0,90 \\
\hline Onyx & CF & 4 & 0,45 \\
\hline Onyx & CF & 12 & 0,90 \\
\hline
\end{tabular}

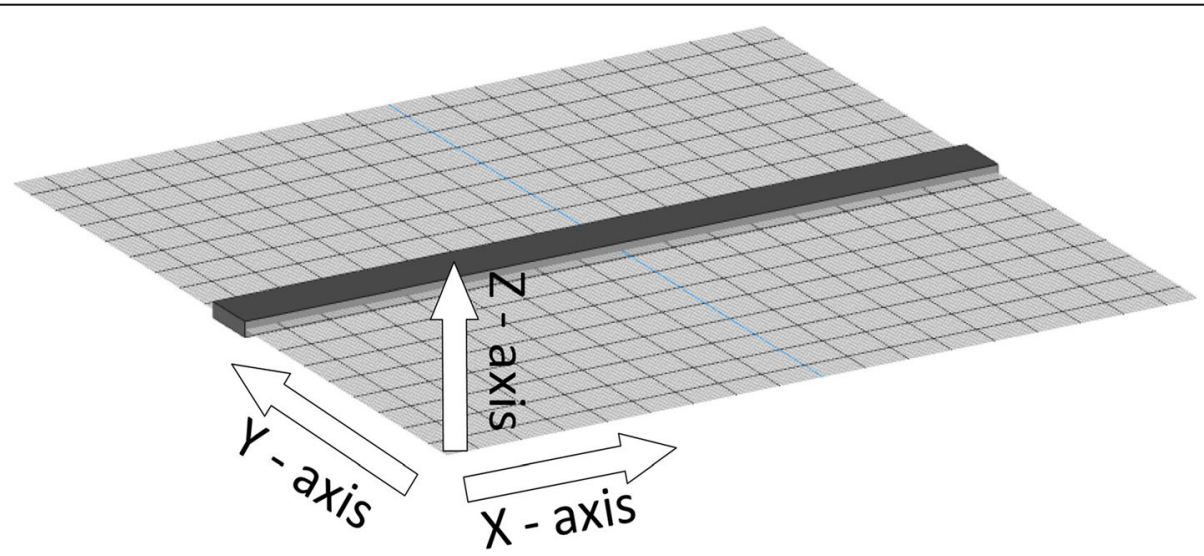

Fig. 2 Build Orientation for Mechanical Test Samples 
Table 3 Tensile and Flexural Test Sample Specifications

\begin{tabular}{lllll}
\hline Test Method & Shape & Length $(\mathbf{m m})$ & Width $(\mathbf{m m})$ & Thickness $(\mathbf{m m})$ \\
\hline Tensile ASTM D3039 & Rectangular & 175 & 12.7 & 3.2 \\
Flexural ASTM 7264 & Rectangular & 150 & 13 & 4
\end{tabular}

Tensile and flexural test specimens were manufactured, based on standard designs; ASTM D3039 [29] for tensile samples and ASTM D7264 [29] for flexural samples (Table 3). ASTM D3039 was chosen over an ISO standard as it allows for easier set-up to avoid flexure of the sample under test [30]. ASTM 7264 (four-point bend testing) was preferred over three-point bend testing as it has been shown that the error in the flexural modulus from 3-point is $5 \%$ higher than 4-point [31]. The specifications of the samples are given in Table 1. ASTM D3039 requires the addition of tabs at the sample ends to avoid damage due to test grips. The standard allows for the printing of the tabs during sample manufacture, as used by Giannakis [32], however, the more widely accepted method of using adhesion of tabs was employed to reduce AM costs and time. Tabs were applied following ASTM D3039, using NEMA standards Grade G-10/FR4 composite (PAR Group Ltd., UK), $30 \mathrm{~mm}$ tab length and a bevel angle of $90^{\circ}$, also recommended by Hodgkinson [30]. The tabs were adhesively bonded to the specimens using a heatcured epoxy VTA 260 (Cytec Industrial Materials (Derby) Ltd., UK). The samples were vacuum bagged and heated at $65^{\circ} \mathrm{C}$ for $16 \mathrm{~h}$ to effect a cure.

Tensile testing was performed at $20-22^{\circ} \mathrm{C}$ using an Instron 3367 tensile testing rig (Instron, USA), with a $30 \mathrm{kN}$ load cell with a cross-head speed of $2 \mathrm{~mm} / \mathrm{min}$ (as per ASTM D3390). An extensometer (Instron, USA) with
$50 \mathrm{~mm}$ gauge length was used. The dimensions of each sample were taken at three places using a digital micrometre screw gauge with measurement accuracy $\pm 0.001 \mathrm{~mm}$.

4-point flexural testing was performed at $20-22^{\circ} \mathrm{C}$ using an Instron 5800R (Instron, USA), with a $100 \mathrm{kN}$ load cell. A cross-head speed of $1 \mathrm{~mm} / \mathrm{min}$ was employed as per ASTM 7264. The span length was set to $128 \mathrm{~mm}$, corresponding to a 32:1 span to thickness ratio as per the standard. Flexural modulus was calculated for the slope of the stress-strain curve at $0.15 \%$ strain level.

\section{Results and discussion}

The data from the mechanical testing was analysed within a statistical analysis software, Minitab v19 (Minitab LLC, USA). An Analysis of Variance was performed to provide the size of each parameter effect ( $F$-Value) and the significance of each parameter effect ( $P$-Value). The results of the statistical analysis for the UTS and E are given in Tables 4 and 5 respectively.

The Pareto charts for the standardised parameter effects for UTS and E are given in Figs. 3 and 4.

As can be seen from Tables 4 and 5, and Figs. 3 and 4, for both UTS and E, all four main parameters are statistically significant (Standard Effect is higher than the significance line for $95 \%$ significance) and can control the mechanical response of the material, although the

Table 4 ANOVA Results for Main Parameters and All Interactions for UTS

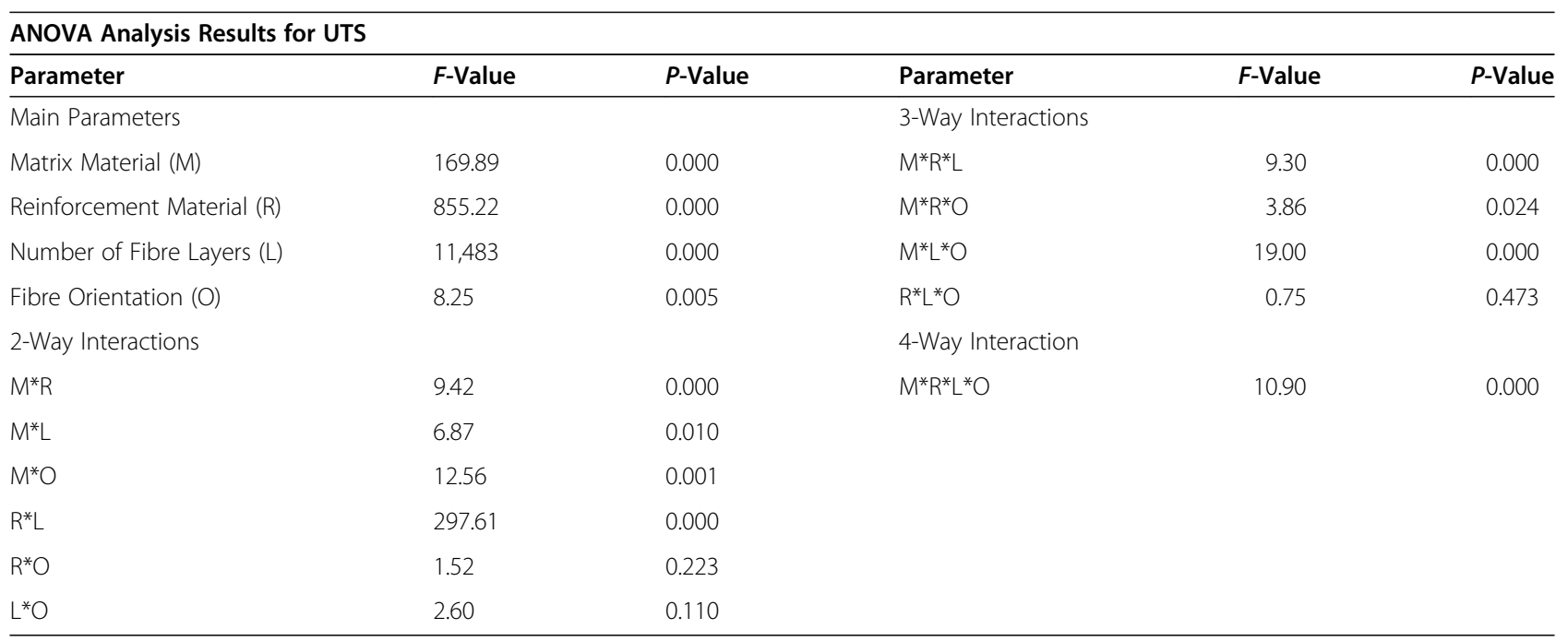


Table 5 ANOVA Results for Main Parameters and All Interactions for E

\begin{tabular}{|c|c|c|c|c|c|}
\hline \multicolumn{6}{|c|}{ ANOVA Analysis Results for E } \\
\hline Parameter & F-Value & $P$-Value & Parameter & F-Value & $P$-Value \\
\hline Main Parameters & & & 3-Way Interactions & & \\
\hline Matrix Material (M) & 6.90 & 0.010 & $M^{*} R^{*} L$ & 33.30 & 0.000 \\
\hline Reinforcement Material (R) & 1338 & 0.000 & $M^{*} R^{*} O$ & 137.46 & 0.000 \\
\hline Number of Fibre Layers (L) & 2733 & 0.000 & $M * L^{*} O$ & 55.09 & 0.000 \\
\hline Fibre Orientation (O) & 96.83 & 0.000 & $R^{*} L^{*} O$ & 8.66 & 0.000 \\
\hline 2-Way Interactions & & & 4-Way Interaction & & \\
\hline$M^{*} R$ & 114.25 & 0.000 & $M^{*} R^{*} L^{*} O$ & 99.16 & 0.000 \\
\hline$M * L$ & 17.63 & 0.000 & & & \\
\hline$M^{*} O$ & 68.73 & 0.000 & & & \\
\hline$R^{*} L$ & 76.28 & 0.000 & & & \\
\hline $\mathrm{R}^{*} \mathrm{O}$ & 64.96 & 0.000 & & & \\
\hline$L^{*} \mathrm{O}$ & 37.52 & 0.000 & & & \\
\hline
\end{tabular}

number of layers exerts the greatest influence on both UTS and E, and is significantly more important than any other parameter. This is as expected since the UTS and $E$ of the reinforcement fibres (Table 1) are at least an order of magnitude greater than those of the polymer matrix materials, and, via the Rules of Mixtures (Eqs. 1 and 2), have increased effect in increasing the mechanical properties as their volume fraction $(f)$, e.g. number of layers, is increased.

It is surprising that the type of reinforcement has such a small effect on the mechanical response, with the Standardised Effect on UTS for 'reinforcement' being only
$27 \%$ of that for 'layers' (Fig. 3), although for E, the effect of reinforcement type is more significant, being $70 \%$ of that for 'layers' (Fig. 4).

The type of matrix material has been shown to have very little effect on the mechanical response, with the Standardised Effect for 'matrix' being only $12 \%$ of that for 'layers' for UTS (Fig. 3) and only 5\% of that for 'layers' for E (Fig. 4). Also, for E, the 'matrix' parameter is only just significant (2.63) compared to the significance limit of 1.98. This is also in agreement with the mechanical performance of the elements forming the composite. UTS for Nylon and Onyx are similar (36 and

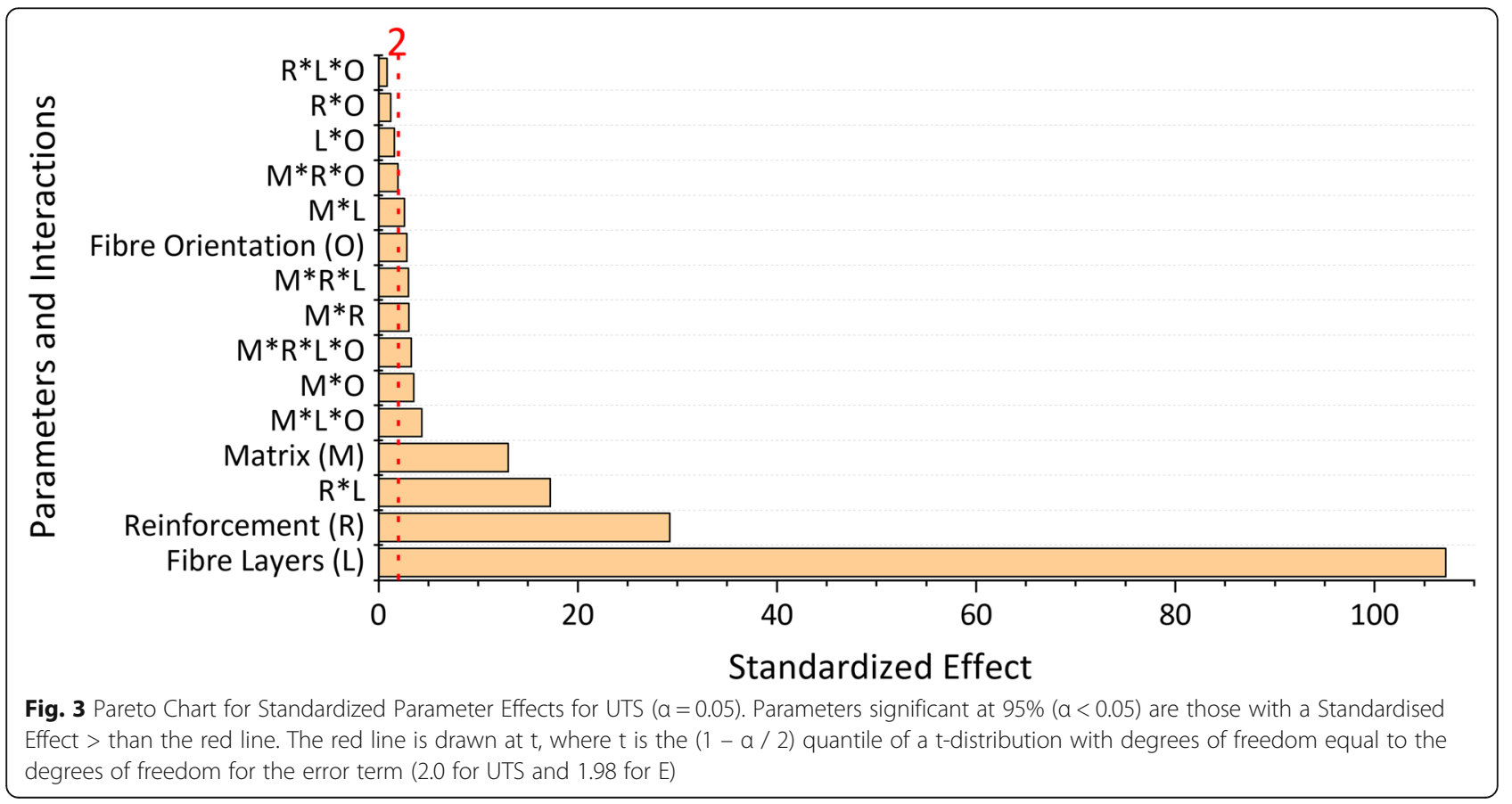




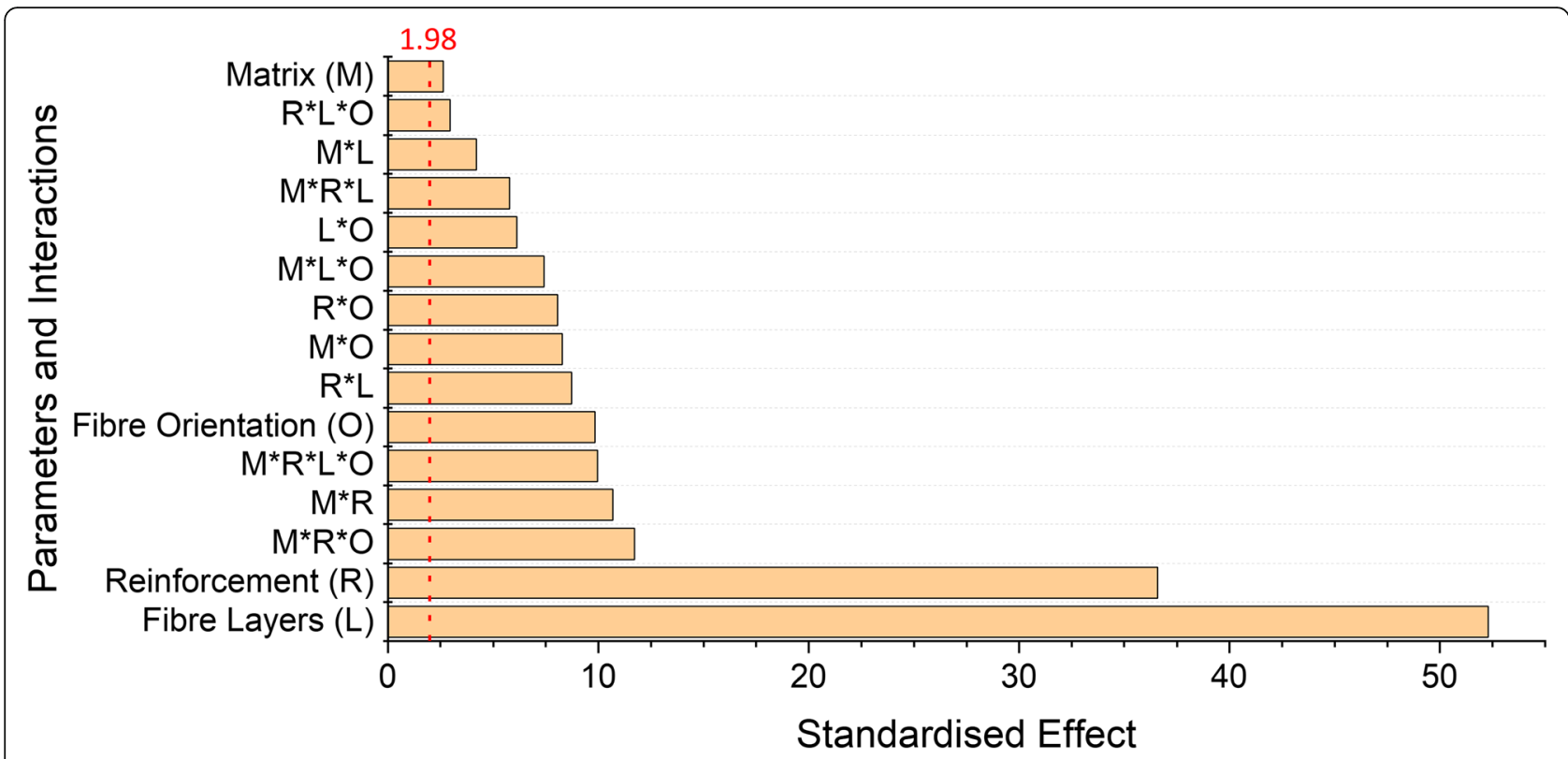

Fig. 4 Pareto Chart for Standardized Parameter Effects for $E(a=0.05)$. Parameters significant at $95 \%(a<0.05)$ are those with a Standardised Effect $>$ than the red line. The red line is drawn at $t$, where $t$ is the $(1-a / 2)$ quantile of a t-distribution with degrees of freedom equal to the degrees of freedom for the error term (2.0 for UTS and 1.98 for E)

$51 \mathrm{MPa}$ ), with Onyx only $42 \%$ higher than Nylon; whereas for $\mathrm{E}$, there is a $157 \%$ increase for Onxy over Nylon (3.4 vs $1.6 \mathrm{GPa})$, hence, the smaller statistical effect on UTS, and larger effect on E, of selecting Onyx over Nylon as the matrix material. Thus, lower-cost, and more abundant Nylon matrix material offers similar performance to the more expensive Onyx.

Fibre orientation, within the bounds of these trials $(0,45$ and 0,90 bi-directional orientations) has very little impact on either UTS (2.87 at significance limit of 2.0) (Fig. 3) or E (9.84 at significance limit of 1.98), being only 3\% (UTS) and 19\% (E) of the standardized effect of 'layers'.

Table 6 below shows the results for the mechanical performance of the highest strength / highest stiffness samples (using 12 layers of reinforcement and $(0,90)$ reinforcement deposition). These are compared to the theoretical values obtained using the Rule of Mixtures (ROM) as given in Eqs. 1 and 2.

$$
\begin{aligned}
& \text { UTS }=\text { fUTS }_{f}+(1-f) \text { UTS }_{m} \\
& E=E_{f} f+(1-f) E_{m}
\end{aligned}
$$

Where $f$ is the fibre volume fraction in the composite, $U T S_{f}$ and $U T S_{m}$ are the UTS of the fibre and matrix respectively, and $E_{f}$ and $E_{m}$ are the flexural moduli of the fibre and matrix respectively.

The selection of parameter levels for main parameters can be informed from the Main Effects Plots (Figs. 5 and 6). These indicate the change in observed average (over all samples) mechanical response as a result in change in parameter level. Tables 7 and 8 show the change in average UTS (as a \% of the global average UTS) and change in average $\mathrm{E}$ (as a \% of the global average $\mathrm{E}$ for

Table 6 Comparison of Experimental Values for UTS and E Against Calculated Values. $\Delta$ is Experimental value as a $\%$ of ROM value

\begin{tabular}{lllllcc}
\hline Composite & UTS (MPa) Experimental & $\begin{array}{l}\text { UTS (MPa) } \\
\text { ROM }\end{array}$ & $\boldsymbol{\Delta}(\%)$ & E (GPa) Experimental & E (GPa) ROM & $\boldsymbol{\Delta}(\%)$ \\
\hline Nylon-GF-12-90 & $220 \pm 4$ & 273 & 81 & $6.4 \pm 0.1$ & 8.3 & 77 \\
Nylon-CF-12-90 & $249 \pm 13$ & 429 & 58 & $13.02 \pm 0.06$ & 21.24 & 61 \\
Nylon-KF-12-90 & $158 \pm 8$ & 282 & 56 & $7.52 \pm 0.07$ & 70.26 & 73 \\
Onyx-GF-12-90 & $212 \pm 5$ & 267 & 79 & $7.3 \pm 0.1$ & 15.3 & 78 \\
Onyx-CF-12-90 & $278 \pm 8$ & 387 & 72 & $15.5 \pm 0.3$ & 10.99 & 72 \\
Onyx-KF-12-90 & $161 \pm 4$ & 276 & 58 & $8.00 \pm 0.07$ & & 73 \\
\hline
\end{tabular}




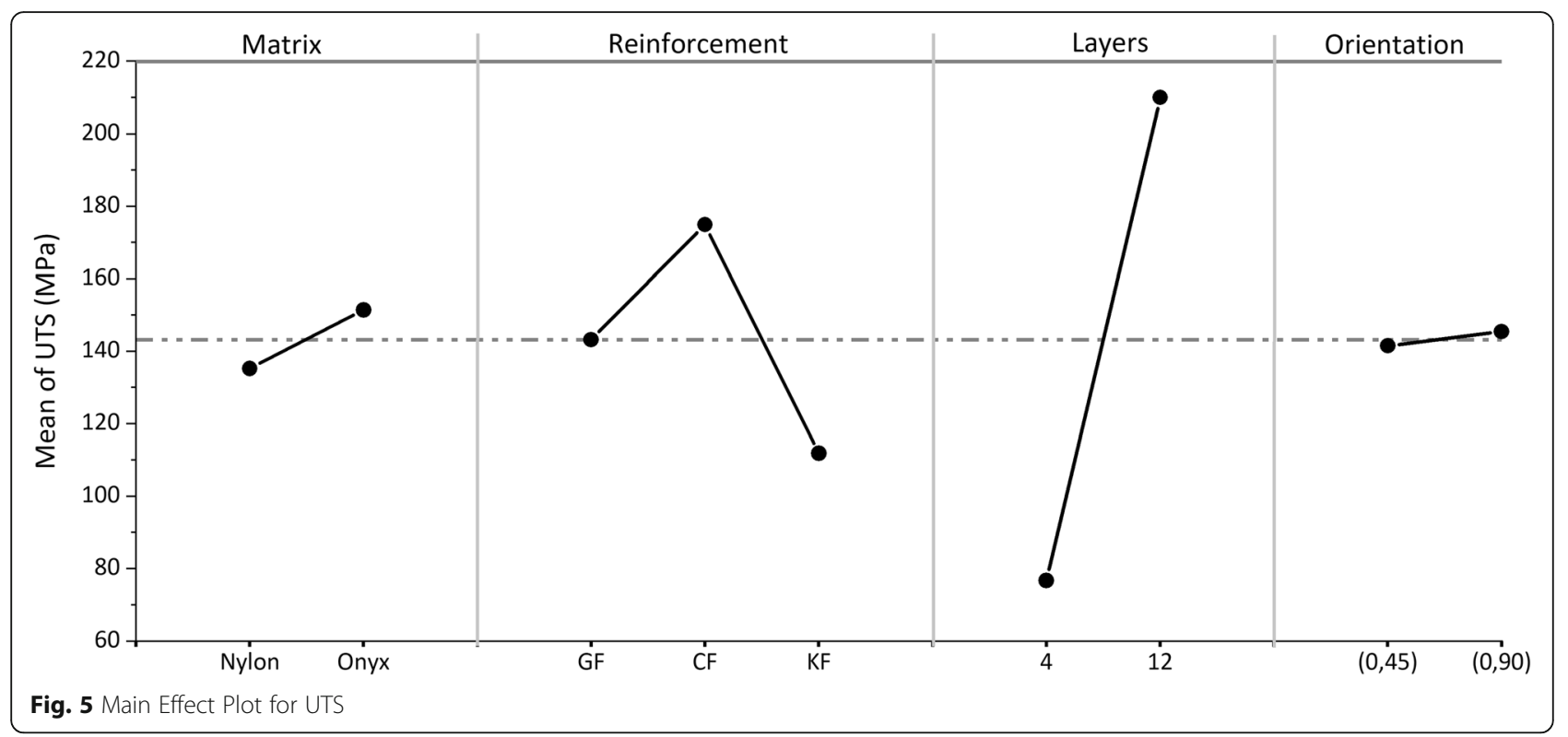

each of the parameter levels. From Figs. 5 and 6 and Tables 7 and 8 , it is clearly evident that the results for UTS and E follow similar, but not identical, trends.

For 'matrix' parameter, selecting Onxy over Nylon does provide a small increase in both UTS and E, but this is only from $5.58 \%$ below global mean to $11.66 \%$ above (UTS) (Table 7) and from $-1.55 \%$ below global mean to $1.55 \%$ above (E) (Table 8 ). This is due to the higher UTS and E values for Onyx over Nylon (Table 1). Thus, optimal properties are achieved by selecting Onyx over Nylon as matrix material, although, as discussed later, the improvements in mechanical response may not outweigh the extra cost incurred.
For 'reinforcement' parameter, there is a large effect in moving from GF to CF, with large increases in both UTS (from $0.14 \%$ below global mean to $22.06 \%$ above Fig. 4 and Table 7) and E (from $0.12 \%$ below global mean to $42.75 \%$ above - Fig. 5 and Table 8). There is a nearly equally sized decrease in properties for both UTS and $\mathrm{E}$ when selecting KF, shifting to $22.94 \%$ below global mean (UTS) and $16.98 \%$ below global mean (E). The increase in properties in selecting CF over both GF and $\mathrm{KF}$ is due to CF having the highest performance of any of the reinforcement materials (Table 1). None of the composites performed to the predicted level (Table 6), with UTS being $58-81 \%$ of predicted and E being $61-$

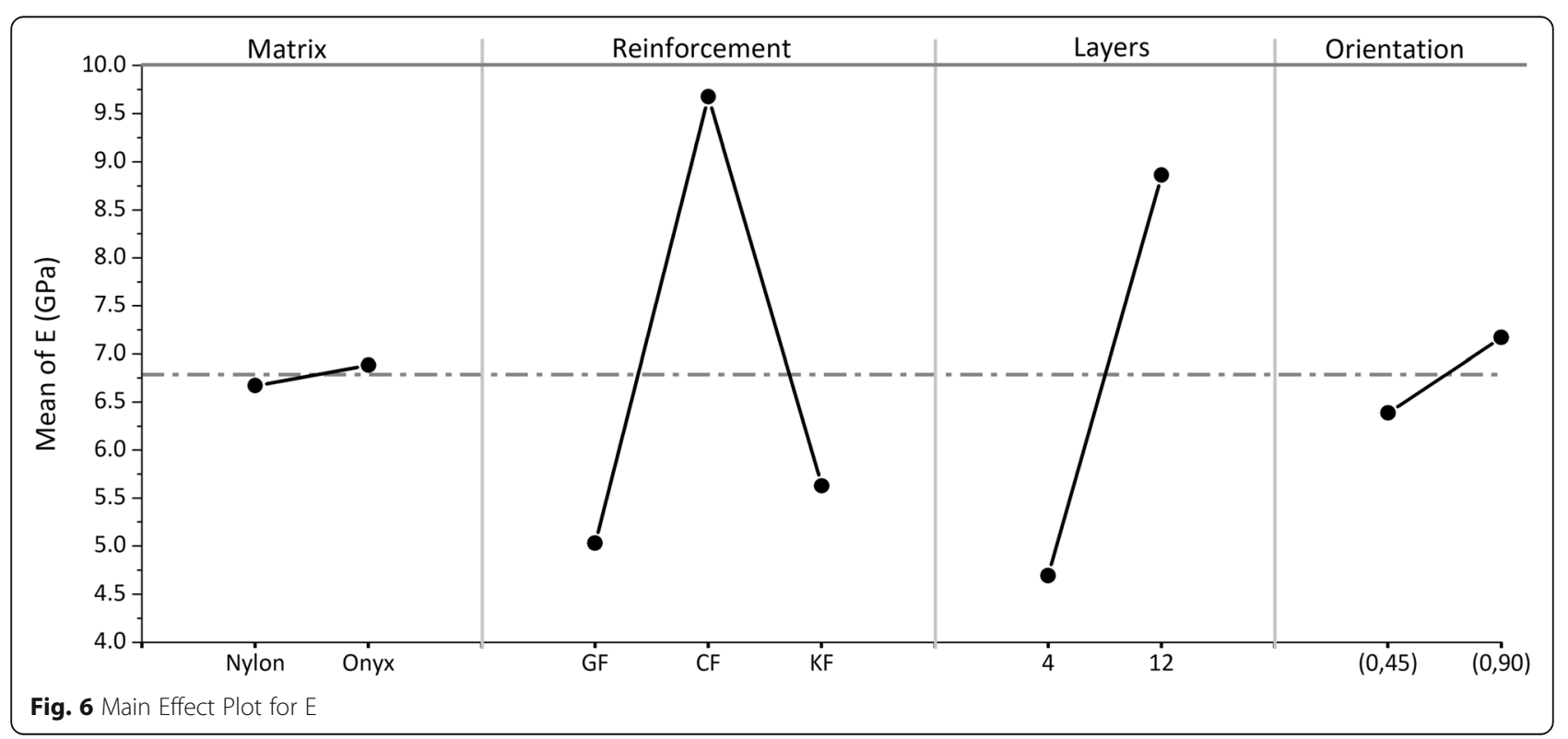


Table 7 The differential mean UTS values (as a \% of the global average UTS - dotted line on Fig. 5)

\begin{tabular}{llllc}
\hline & \multicolumn{3}{l}{ Parameter } & \\
\cline { 2 - 5 } Level & Matrix & Reinforcement & Layers & Orientation \\
\hline $\mathbf{1}$ & $-5.68 \%$ & $-0.14 \%$ & $-46.52 \%$ & $-1.23 \%$ \\
$\mathbf{2}$ & $11.66 \%$ & $22.06 \%$ & $46.56 \%$ & $1.27 \%$ \\
$\mathbf{3}$ & & $-22.94 \%$ & & \\
\hline
\end{tabular}

$78 \%$ of predicted. The lower performance agrees with previous work by Dickson et al. [12], where the reduced performance was concluded to be due to failure of the bond between the fibre and matrix, leading to fibre pullout. The poor performance of KF over GF, despite its higher mechanical properties over GF (Table 1), also agrees with the findings of Dickson et al. [12], where fibre pull-out was more prevalent for $\mathrm{KF}$, and, due to the pulled fibres being residue free, are thought to be weaker bonded to the matrix than CF or GF, which both left residue bonded to the fibres. Thus, optimal properties are obtained by selecting $\mathrm{CF}$ as reinforcement material.

The 'layers' parameter has the largest influence over the mechanical properties. From the Main Effect plots for UTS and E (Figs. 5 and 6), moving from 4 layers to 12 layers provides an improvement in UTS from $46.52 \%$ below global mean to $46.56 \%$ above global mean; the same change results in raising $E$ from $30.76 \%$ below global mean to $30.76 \%$ above global mean. The inability to attain the predicted performance values (Table 6), may also be due to increasing porosity levels observed between fibre and matrix for increasing fibre fraction [12], thus limiting the effectiveness of increasing fibre content (number of layers). Thus, optimal properties are obtained by using a higher number of reinforcement layers, and, as we see later, is an affordable choice.

The Main Effect plots also show a marginal improvement in moving from a $(0,45)$ pattern to a $(0,90)$ pattern, with UTS increasing from $1.23 \%$ below global mean to $1.27 \%$ above global mean (Table 7), and E increasing from $5.78 \%$ below global mean to $5.80 \%$ above global mean (Table 8). This small effect may be due to the similarity between the two lay-up patterns. Higher performance was achieved by Klift et al. [23], achieving $400 \mathrm{MPa}(\sigma=20.35)$

Table 8 The differential mean E values (as a \% of the global average $E$ - dotted line on Fig. 6)

\begin{tabular}{llllc}
\hline & \multicolumn{3}{l}{ Parameter } & \\
\cline { 2 - 5 } Level & Matrix & Reinforcement & Layers & Orientation \\
\hline $\mathbf{1}$ & $-1.55 \%$ & $-0.12 \%$ & $-30.76 \%$ & $-5.78 \%$ \\
$\mathbf{2}$ & $1.55 \%$ & $42.75 \%$ & $30.76 \%$ & $5.80 \%$ \\
$\mathbf{3}$ & & $-16.98 \%$ & & \\
\hline
\end{tabular}

UTS for Nylon-Carbon samples, using a concentric ring lay-up, with our Nylon-CF only achieving $249 \pm 6 \mathrm{MPa}$. Although the results are not directly comparable as Klift et al. [23] used an increased number of reinforcement layers of 16, compared to 12 in our research. It is clear though from this research that a $(0,90)$ pattern does help to optimise the UTS and E and has no significant effect on the cost (as we see later).

The tensile and flexural stress-strain behaviour for the six composite material combinations (for the highest UTS samples (12 layers and 0,90 lay-up orientation) are given in Figs. 7 and 8. It can be seen that the tensile and flexural properties are determined primarily from the reinforcement type. The failure behaviour under tensile load is similar for all material combinations, with fairly constant modulus up to the failure point and then rapid failure. This is due to the UTS of the fibres being significantly higher than that of the matrix materials (Table 1), so once fibre failure occurs, the matrix fails very rapidly. Both the CF and GF failure occurs at around the nominal strain (1.5 and $2.1 \%$ respectively), but the KF samples fail below their nominal value (2.7\%), failing at $1.9 \%$, supporting further that the failure mechanism is a combination of fibre breakage and fibre pull-out as described earlier. This is further supported by the behaviour of the KF reinforced samples under compressive load (Fig. 8), having a lower flexural modulus than GF samples at higher strain, despite the nominally higher modulus (Table 1 ).

The Cost to Strength Ratio ( $\$ / \mathrm{MPa}$ ) for UTS, and UTS values for each of the 24 composites and for the un-reinforced Nylon and Onyx (average of 5 samples for each) is given in Fig. 9. The Cost Ratio ( $\$ / \mathrm{GPa}$ ) for $\mathrm{E}$, and $E$ values for each of the 24 composites and for the un-reinforced Nylon and Onyx (average of 5 samples for each) is given in Fig. 10. The cost for manufacture used to calculate the Cost Ratio values was calculated using the material usage (matrix and reinforcement) recorded from the Mark Two printer after each build (and divided by 5 to obtain cost / part).

It can be deduced from the analysis of cost verses strength (Fig. 9) and stiffness (Fig. 10) that the lowest cost to strength ratio (most desirable) is achieved using a GF reinforced Nylon with 12 layers and $(0,90)$ lay-up, at $0.0221 \pm 0.0002 \$ \mathrm{MPa}^{-1}$, and achieving $220 \pm 2 \mathrm{MPa}$ UTS $(79 \pm 1 \%$ of highest UTS, achieved using CF reinforced Onyx with 12 layers and $(0,90)$ lay-up. The equivalent material using $(0,45)$ only achieves a UTS of $187 \pm 4 \mathrm{MPa}(67 \pm 1 \%$ of highest UTS $)$ at a cost to strength ratio of $0.0261 \pm 0.0005 \$ \mathrm{MPa}^{-1}$. The highest performing composite, $278 \pm 4 \mathrm{MPa}$ (CF reinforced Onyx with 12 layers and $(0,90)$ lay-up) has a cost to strength ratio of $0.0303 \pm 0.0004 \$ \mathrm{MPa}^{-1}, 37 \pm 2 \%$ higher cost than the optimum cost to strength ratio material. Thus, for a $21 \pm 1 \%$ increase in strength, a $37 \pm 2 \%$ increase in cost is incurred, 


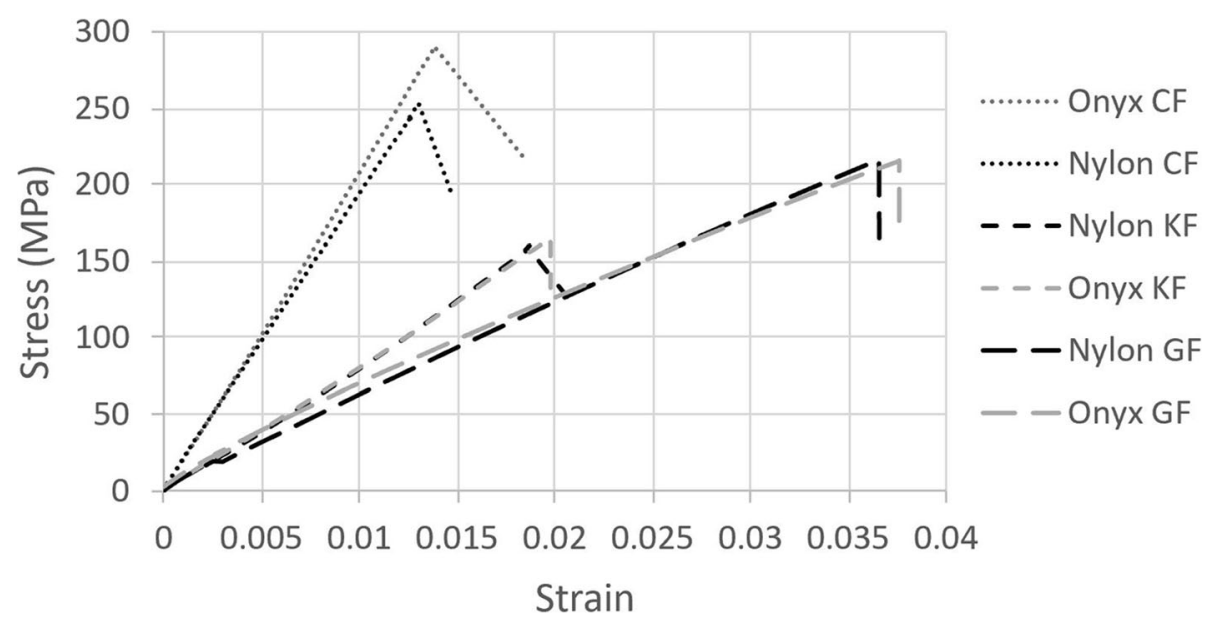

Fig. 7 Tensile Stress-Strain Curves for Highest Performing Composites (12 layers, 0,90 orientation)

making the most cost-effective material option highly attractive for all but the most demanding applications.

The stiffest material is CF reinforced Onyx with 12 layers and $(0,90)(15.5 \pm 0.3 \mathrm{GPa})$, and is 2 nd most costeffective material $\left(0.49 \pm 0.06 \$ \mathrm{GPa}^{-1}\right)$ (Fig. 10). The most cost-effective material is GF reinforced Nylon with 12 layers and $(0,45)\left(0.40 \pm 0.02 \$ \mathrm{GPa}^{-1}\right)$, which is $20 \pm$ $3 \%$ lower cost but only retains $39 \pm 2 \%$ of the stiffness of the stiffest material. It is therefore only practical to use this most cost-effective material where high stiffness is not a design requirement. Only three materials have $\mathrm{E}>$ $10 \mathrm{GPa}$, and these are all CF reinforced with 12 layers. It is therefore practical to use the stiffest material (CF reinforced Onyx with 12 layers and $(0,90)$ ) for all but the most cost-sensitive applications. KF does not provide any technical advantage over CF and is also not competitive economically.

\section{Conclusions}

The mechanical capabilities of a continuous fibre thermoplastic composite AM technology (Mark Two, MarkForged Inc., USA) has explored for all the continuous-fibre reinforcement options available (glass, carbon and $\mathrm{Kevlar}^{\odot}$ ) and have demonstrated that it has the capability to engage in engineering applications provided the correct choice of process (printing) parameters and materials is made.

The effect of the key process parameters of: matrix polymer, reinforcement fibre, level of reinforcement and reinforcement lay-up, upon the strength (UTS) and stiffness (E) were evaluated using a DoE and ANOVA methodology, per approved ASTM standards. 'Layers' and 'Reinforcement' play a very significant role in determining both tensile and flexural properties, with Layers being significantly more important in determining UTS

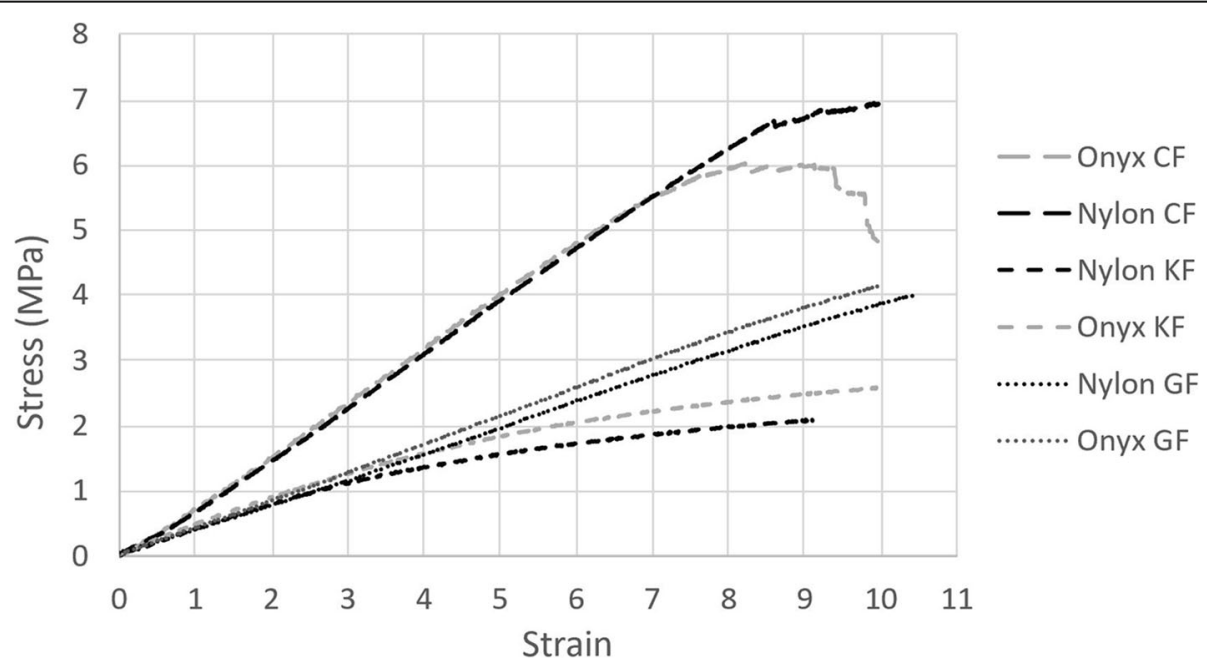

Fig. 8 Flexural Stress-Strain Curves for Highest Performing Composites (12 layers, 0,90 orientation) 


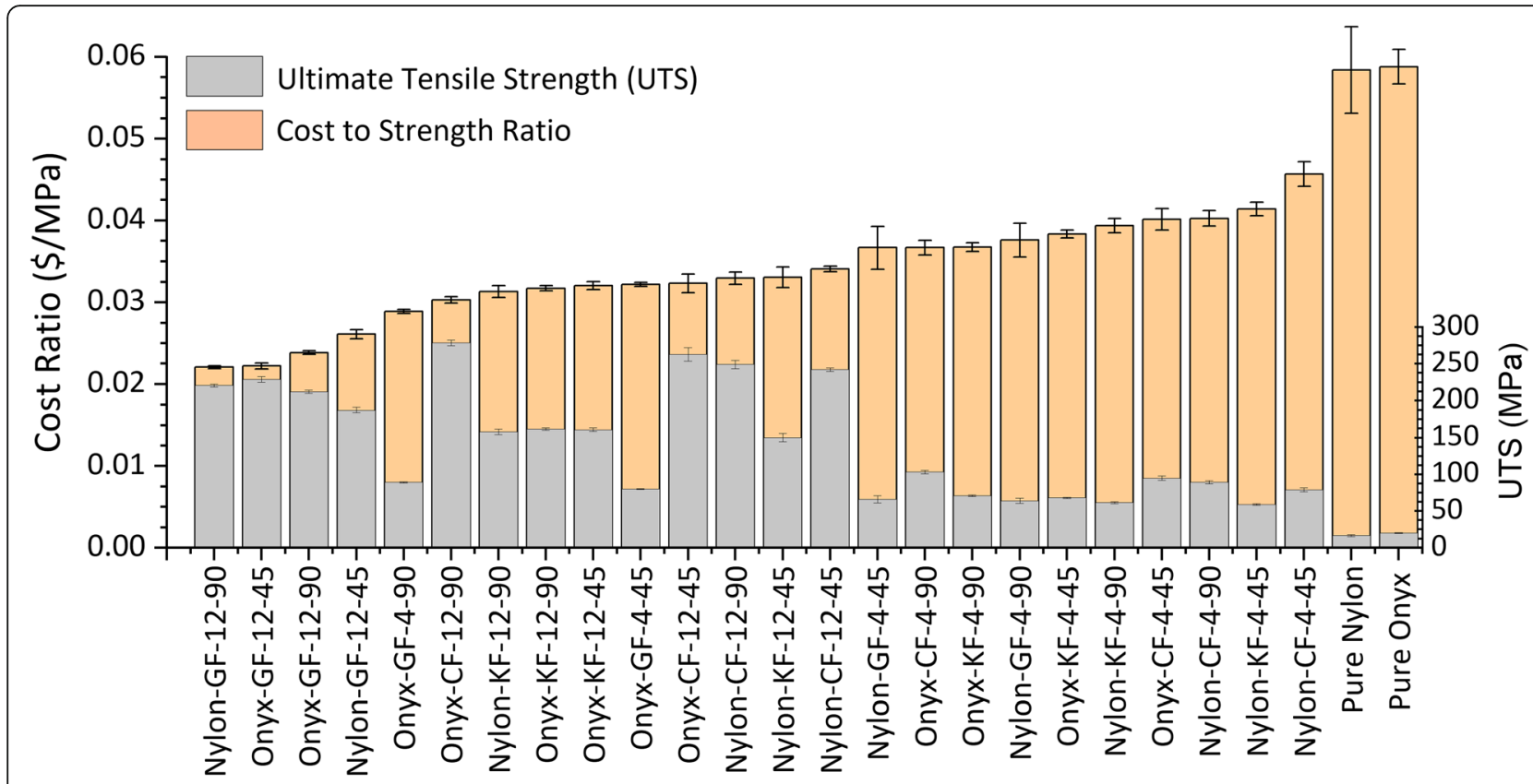

Fig. 9 Cost Ratio (\$/MPa) and UTS for Composites and Un-reinforced Materials

than any other parameter (73\% higher standardized effect than 'reinforcement').

The fibre layup angle and matrix material have a minor effect on the mechanical properties. 'Matrix' having a standardized effect only $12 \%$ that of 'layers' for
UTS and 5\% that of layers for E; 'orientation' having a standardized effect only $3 \%$ that of 'layers' for UTS and $19 \%$ that of layers for E.

Optimal properties are obtained by selecting CF as reinforcement material, using a higher number of

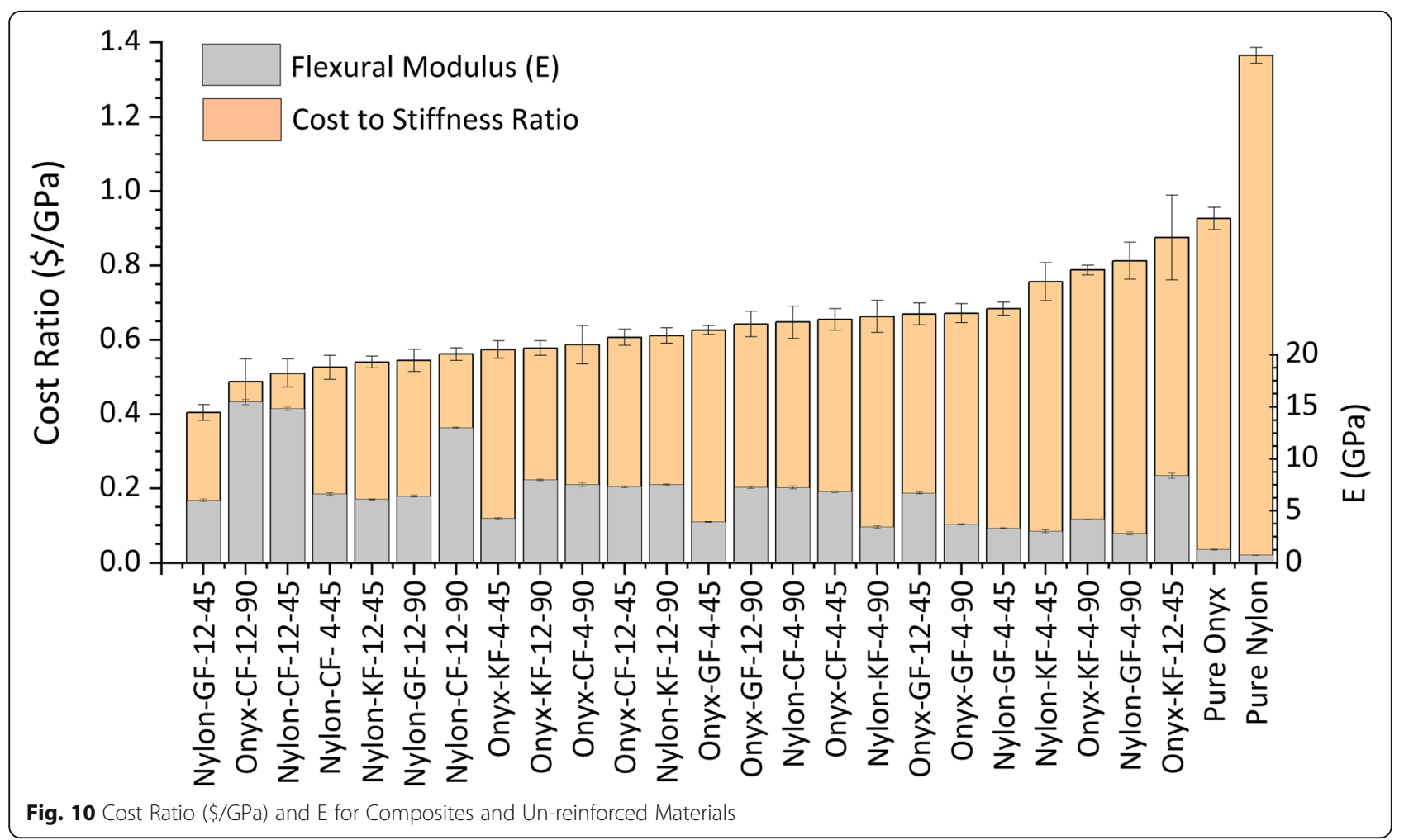


reinforcement layers (demonstrated to be an affordable choice) and by selecting Onyx over Nylon as matrix material, although the improvements in mechanical response may not outweigh the extra cost incurred.

For UTS, the size of effect of 'reinforcement' was found to be $\mathrm{CF}>\mathrm{GF}>\mathrm{KF}$; while for $\mathrm{E}$ it was $\mathrm{CF}>\mathrm{KF}>$ GF. GF can improve the tensile and flexural modulus of Nylon by $1340 \pm 293 \%$ and $876 \pm 52 \%$ and of Onyx by $1390 \pm 234 \%$ and $569 \pm 37 \%$. CF can improve the tensile and flexural modulus of Nylon by $1472 \pm 187 \%$ and $2024 \pm 107 \%$ and of Onyx by $1400 \pm 153 \%$ and $1214 \pm$ $81 \%$. KF can improve the tensile and flexural modulus of Nylon by $960 \pm 243 \%$ and $1027 \pm 53 \%$ and of Onyx by $810 \pm 83 \%$ and $627 \pm 37 \%$.

The highest UTS $(278 \pm 8 \mathrm{MPa})$ and stiffest material $(15.5 \pm 0.3 \mathrm{GPa})$ is CF reinforced Onyx with 12 layers and $(0,90)$ The stiffest material is CF reinforced Onyx with 12 layers and $(0,90)$. The best cost-strength ratio is obtained using a GF reinforced Nylon with 12 layers and $(0,90)$ layup, achieving $220 \pm 2 \mathrm{MPa}$ UTS (79 $\pm 1 \%$ of highest UTS). The best cost-stiffness ratio is obtained using a GF reinforced Nylon with 12 layers and $(0,45)$, achieving $6.03 \pm$ $0.1 \mathrm{GPa}(39 \pm 2 \%$ of the stiffest material).

\section{Abbreviations}

ABS: Acrylonitrile Butadiene Styrene; AM: Additive Manufacturing; ANOVA: Analysis of Variance; CF : Carbon Fibre; CNC: Computer Numerical Control; E: Flexural Modulus; GF: Glass Fibre; GPa: Giga Pascals; KF: Kevlar ${ }^{\oplus}$ Fibre; kN: Kilo Newton; mm: Millimetres; MPa: Mega Pascals; PLA: Polylactic Acid; PP: Polypropylene; SWNTs: Single Wall Carbon Nanotubes; ROM: Rule of Mixtures; UTS: Ultimate Tensile Strength; VGCFs: Vapour Grown Carbon Fibres

\section{Acknowledgements}

The authors wish to thank WMG, University of Warwick, UK for supporting the MSc in Materials and Manufacturing under which this research was performed.

\section{Authors' contributions}

F Dantas - Performed research and developed report for MSc. Data analysis. K Couling - Helped in manufacture and test of samples. G Gibbons -

Primary author of paper, data analysis. The authors read and approved the final manuscript.

\section{Funding}

WMG, University of Warwick.

\section{Availability of data and materials}

All data is stored on a secure server at the WMG, University of Warwick.

\section{Competing interests}

None.

Received: 29 May 2020 Accepted: 15 September 2020

Published online: 21 September 2020

\footnotetext{
References

1. C. Zweben, Mech. Eng. Handbook, Vol. 1 Mater. Eng. Mech, 4th edn. (Wiley, Chichester, 2015), pp. 401-438

2. J. Go, S. Schriffres, A. Stevens, A. Hart, Addit. Manuf. 16, 1 (2017)

3. R. S, F. R, in Adv. Compos. Mater. Aerosp. Eng, 1st edn., ed. by R. Sohel, F. Raul. (Woodhead Publishing, Witney, 2016), pp. 1-15

4. T. Johnson, (n.d.)

5. J. Hale, (2006)

6. H. Ning, G. Janoswki, U. Vaidya, G. Husman, Compos. Struct. 80, 82 (2007)
}

7. Anon, (2018)

8. J. Plocher, A. Panesar, Matrials Des. (2019)

9. M. Oyesola, K. Mpofu, N. Mathe, in 2nd Int. Conf. Sustain. Mater. Process. Manuf. SMPM 2019, ed. by A. Shih. (Procedia Manufacturing, Sun City, 2019), pp. $155-163$

10. J. Steuben, D. Bossuyt, C. Turner, ASME Int. Des. Eng. Tech. Conf. Comput. Inf. Eng. Conf (ASME, Boston, 2015)

11. Anon, (2020)

12. A. Dickson, K.-A. Ross, D. Dowling, Compos. Struct. 206 (2018)

13. Anon, ISO/ASTM52900-15 Standard Terminology for Additive Manufacturing - General Principles - Terminology (2015)

14. M. Leary, Design for Additive Manufacturing, 1st edn. (Elsevier, London, 2020)

15. M. Ziaee, N. Crane, Addit. Manuf. 28, 781 (2019)

16. X. Shen, H. Naguib, Addit. Manuf. 29 (2019)

17. A. Kamper, J. Triebs, S. Kawollek, P. Ayvaz, S. Hohenstein, Procedia CIRP 81, 815 (2019)

18. C. Chua, K. Leong, C. Lim, Rapid Prototyping: Principles and Applications, 3rd edn. (World Scientific Publishing Co. Pte. Ltd, Singapore, 2010)

19. O. Carneiro, A. Ferreira da Silva, R. Gomes, Mater. Des. 83, 768 (2015)

20. M. Shofer, F. Rodrigues-Marcias, E. Barrera, K. Lozano, J. Rodri, Appl. Polym. Sci. 89, 3081 (2003)

21. R. Matsuzaki, M. Ueda, M. Namiki, T.-K. Jeong, H. Asahara, K. Horiguchi, T. Nakamara, A. Todoroki, Y. Hirano, Sci. Rep. (2016)

22. K.-I. Mori, T. Maeno, Y. Nakagawa, Procedia Eng. 81, 1595 (2014)

23. F. Van der Klift, Y. Koga, A. Todoroki, M. Ueda, Y. Hirano, R. Matsuzaki, Open J. Compos. Mater. 6, 18 (2016)

24. A. Khatri, Effect of Manufacturing-Induced Defects and Orientation on the Failure and Fracture Mechanism of 3D Printed Structures (The University of Texas at Arlington, Arlington, 2016)

25. F. Ning, W. Cong, Y. Hu, H. Wang, J. Compos. Mater. 51, 451 (2016)

26. Markforged, (n.d.)

27. Markforged, (2019)

28. S.-H. Ahn, M. Montero, D. Odell, S. Roundy, P. Wright, Rapid Prototyp. J. 8 248 (2002)

29. Anon, Stand. Test Method (n.d.)

30. J. Hodkinson, Failure Mechanisms in Polymer Matrix Composites, 1st edn. (Woodhead Publishing, Witney, 2012)

31. F. Mujika, Polym. Test. 25, 214 (2006)

32. E. Giannakis, Mechanical Properties of $3 D$ Printed Specimens (International Hellenic University, Thermi, 2018)

\section{Publisher's Note}

Springer Nature remains neutral with regard to jurisdictional claims in published maps and institutional affiliations.

\section{Submit your manuscript to a SpringerOpen ${ }^{\circ}$ journal and benefit from:}

- Convenient online submission

- Rigorous peer review

- Open access: articles freely available online

- High visibility within the field

- Retaining the copyright to your article

Submit your next manuscript at $>$ springeropen.com 